\title{
Application of RFID Technology in Building Intelligent Logistics System in Internet of Things
}

\author{
Guofang Kuang ${ }^{1, a^{*}}$ and Yingcun Cao ${ }^{1}$ \\ ${ }^{1}$ Luoyang Normal University, Luoyang, 471934, China \\ akuangguofang2012@163.com
}

Keywords: Intelligent logistics system; RFID; Internet of things; Electronic commerce; Vehicle

\begin{abstract}
This paper firstly introduces the whole framework of security RFID system. Then, this paper analyzes the functions of the existing logistics system, and points out the defects of the existing system. Intelligent logistics system is concept proposed recently in the intelligent transportation system (ITS) basis and related information technology, electronic commerce (EC) of modern logistics service system operation. The paper presents application of RFID technology in building intelligent logistics system in Internet of things.
\end{abstract}

\section{Introduction}

RFID radio frequency identification technology (Radio Frequency Identification) is an automatic identification technology from the nineteen eighties to mature. It uses the way of non-contact radio frequency two-way channel to exchange data in order to identify the objective. Compared with the traditional magnetic card, IC card, RF card advantage in non-contact, without manual intervention so complete identification work, is suitable for the realization of automation system and is not easy to damage, can identify the high-speed moving objects and can also identify multiple RF card, convenient and quick operation.

In the intelligent logistics, object labeling or user identity is the most important information. Generally speaking, a commodity in the logistics, we must first solve a "who I am" problem. The scholars also had this kind of consciousness, such as new IPv6 protocol theory can give the earth every mote in an IP address [1]. Since the core network information platform of next generation Internet of things will be the Internet, then, every object in the Internet of things, member's identity, whether can also like IP address that, to establish a unified data format, a complete analytical framework, a comprehensive coverage of the region.

The RFID measurement system process: issuing sampling purchase personnel set coal, in the system manufacturers, coal delivery date, batch to RFID card issuing operations, namely card initialization. Is initialized after the card is used for the original notice is issued with coal. Sampling the driver gets card, card sampling, sampling by the sampling personnel, with the input number monitoring system, in sampling system automatically from the card in the middle sampling tank number information, and sent to the computer sampling machine, automatic tank for implementation of sampling machine.

With the wide application of the continuous development of RFID technology and RFID system based on Internet of things in the traditional network, on the basis of existing increased processing platform sensor network and intelligent, the traditional network security measures have been unable to provide reliable security, thus the emergence of new safety hidden danger. The hidden dangers of 2 aspects of RFID system mainly there are privacy and authentication: privacy is mainly to prevent illegal tracking an attacker to any form of RFID tags; in authentication is to make sure that the label can only communicate with a legitimate reader. The paper presents application of RFID technology in building intelligent logistics system in Internet of things. 


\section{Whole Framework of Security RFID System}

Basic RFID system consists of 3 main parts, the label: label in identifying objects, carrying the target identification data, is the real data carrier RFID system, by coupling components and microelectronics chip (including modulator, code generator, clock and memory) group.

The communication between RFID card reader and tag and energy sensing mode can be roughly divided into, inductive coupling (Inductive Coupling) and backscattering coupling (Backscatter Coupling) two, general low-frequency RFID mostly use the first type, and the higher frequency is mostly used in second ways [2]. Reader according to the structure and techniques used in different can be read or read / write device, RFID system information control and processing center. The reader usually consists of a coupling module, receiving module, control module and the interface unit. Half duplex communication by the exchange of information between the reader and transponder, and the reader through the coupling to passive transponder provides energy and timing.

In this paper, the hardware and software of the reader and the tag information is the main threat to the RFID network system security. Application of RFID technology in the system general design, usually attack methods are: information, forgery, tampering with information, information playback interruption of information, as well as the illegal tracking tags, interferes with the normal work of the reader and the tag, the interception of tag data and information transmission. To avoid the hidden trouble of the strategy, avoid the hidden trouble strategies are: Kill command, active jamming, the electrostatic shielding and other physical methods; Hash lock, Hashi, re encryption mechanism, challenge response mechanism, security protocol; using the method, as is shown by equation(1) [3].

$$
\Psi(m, s):=[\overbrace{0, \cdots, 0,}^{s-1} C((m-1) M+s), \overbrace{0, \cdots, 0}^{M-s}]
$$

RFID is used for anti-counterfeiting process consists of many applications, including equipment, software and middleware, labels etc.. This is used for the whole framework of security RFID system, we can see the electronic label began from manufacturers to distributors, and then to the electronic label last retailers with electronic tags to read and write, and finally obtain information goods tracking.

Collaborative or integration is another manifestation of the future logistics. No doubt, the Internet of things will be the logistics enterprises to achieve the best platform for collaborative development. With this platform support, logistics enterprises to realize the seamless connection between the upstream and downstream enterprises, realize the dream of years of capital flow, logistics, information flow of the three in one, so the electronic commerce, joint distribution, the globalization of production, these people look forward to advanced business operation mode, is expected to one.

Random Hash-Lock protocol: mechanisms using a challenge response based on random numbers; Hash chain protocol: is a challenge response protocol based on a shared secret, if 2 different hash reader authentication sponsor, label will send different responses, is an independent ID update ability of active tags; ID change the hash based on: similar to Hash chain protocol, the system uses a random number of feet to label the ongoing dynamic refresh, each response in the ID exchange of information is not the same, can resist the replay attack; Digital Library: David RFID protocol with pre shared secret pseudo-random function is realized based on the distributed RFID query authentication; response authentication protocol: Authentication Protocol is suitable for distributed database environment, is a mutual authentication protocol typical; LCAP protocol is the same: a challenge response protocol, but other challenge response protocol, and in front of the different, the agreement after each execution to dynamically refresh tag ID. Protection of Hash authentication key lock and it is random Hash lock, Hash chain, and Key value renewal random Hash lock, as is shown by equation (2).

$$
M I_{F A}(f, a)=\sum_{f, a} P_{F A}(f, a) \ln \frac{P_{F A}(f, a)}{P_{F}(f) P_{A}(a)}
$$


Reader: reader device used to read or read / write tag data, a radio frequency module (transmitter and receiver), the control unit, and label connected coupling unit [4]. Server: server containing the database processing system, storage and management of relevant information such as labels, label, reader, reading time. The background server receives from a trusted reader to obtain labeled data, data input to its own database, and provides access to label data number.

Comprehensive and supporting technical innovation products again for the honor of national library provides the RFID solution and the success implementation, added beautiful scenery for the construction of new China's largest library [5]. The National Library for RFID tag has three types, including books, private label, CD label; special shelves plate special labels, librarian workstation system, labeled transition system, self-service system, self-help book system, library collection check system, intelligent navigation and retrieval system, security door system.

\section{Development of Intelligent Logistics in Internet of Things}

Logistics should be the first application of the Internet of things, to some extent, can be said to be the main field of application in Internet of things. Many of the emerging technology is first used in military, reflected the role of logistics in military logistics system by. As the world's largest military power, the U.S. military has spared no effort to logistic construction [6].

Although the army logistics support level already leads the world, but to support modern war or stretched. The US Military Logistics Department of transportation the pile up like a mountain hand equipment during the Gulf War, but the management of materials is always a big problem, also appeared in the absence of effective and accurate management situations, tens of thousands of material container because the waybill is lost or damaged, had to open the $75 \%$ container to learn which stored articles.

EPC is Global. It defines and vigorously promote the electronic product code (Electronic Product Code, EPC) is an automatic marking system of Internet of things. Another is IS0 and the standard series ISo 18000. For details on the EPC and IS0 about RFID standards existing in the previous paper, this section will take EPC logistics network architecture as an example, the development of model identification and information service of intelligent logistics. EPC is the predecessor of the Auto-ID center, as is shown by equation (3).

$$
H=E(\Delta I)=-\sum_{i=1}^{n} p_{i} \log \left(p_{i}\right)
$$

Intelligent logistics is the use of integrated intelligent technology, make the logistics system to mimic human intelligence, thinking, perception, learning, inference and solve some problems in their ability of logistics. Intelligent large operations and decision in the logistics process; logistics management as the core, realize the logistics process of storage, transportation, packing, loading and unloading of hierarchical integration and intelligent logistics system, through four intelligent mechanism of intelligent logistics system, namely intelligent information technology, as is shown by equation(4), where $\mathrm{x}$ is intelligent transmission technology, intelligent processing technology.

$$
\bar{x}=W x=\left[\begin{array}{c}
x(-s \max , 1) \\
\delta x(-s \max , 1) \\
\vdots \\
\delta x\left(-1, \frac{k+N}{2}\right)
\end{array}\right]_{m N \times 1}
$$

Intelligent logistics system (ILS) is a concept proposed recently, can think of ILS in the intelligent transportation system (ITS) basis and related information technology, electronic commerce (EC) of modern logistics service system operation [7]. It solves the real-time information acquisition and logistics operation by ITS and related information technology, and in an integrated environment for the acquisition of information analysis and processing, through the information transmission in each 
link in the logistics, to provide detailed information and advisory services for the logistics service providers and customers

Accurate logistics requirements are to minimize the cost and zero waste. Specifically, in the intelligent logistics system in the future, because of widespread use of complex intelligent information processing system and intelligent equipment, logistics enterprise managers hope the precise management of procurement, storage, transfer, assembly, transportation, etc, the inventory, transportation, manufacturing cost to a minimum, at the same time the link may produce waste is reduced to zero.

\section{Application of RFID Technology in Building Intelligent Logistics System in Internet of Things}

Technology of intelligent accesses to make the logistics from passive to active, the realization of active information in logistics process, active monitoring vehicle and goods, active analysis of information, makes the goods began to be implemented tracking and management from the source.

In order to make the vehicle information moving into operation of the logistics chain of information, you need to use a mobile information system [8]. The system and logistics information center constitute a unified whole. To determine the contract data, data transport route, vehicle data and running data need to be collected, storage, exchange and processing [9]. The freight vehicle into the main means of the information chain which is arranged in the vehicle (portable) user computer or specially developed information is mainly large multinational logistics enterprises and logistics enterprises, with the decline in technology updates and information costs, many small and medium-sized logistics enterprises are more and more interested in mobile information technology, as is shown by equation (5).

$$
W_{X} X(m+1)=W_{X} \Phi(m) x(m, M)+W_{X} \bar{w}(m)
$$

Vehicle control system is an important part of operation management of freight vehicle in modern logistics system. Vehicle control technology is to provide logistics support system in the transportation of supply chain of the whole process management function [10]. It includes several aspects of operating freight fleet management, transportation management, freight vehicle electronic clearance, operation of freight vehicle transportation management, dynamic load, vehicle safety monitoring, vehicle safety, vehicle maintenance, transportation of dangerous goods management and other functions. These functions can be simplified, such as registration of vehicle technical performance, size and other formalities, goods distribution, optimization of backhaul information, improve the reliability and efficiency of distribution operations, greatly improve the efficiency of transportation and production, and reduce delays, as is shown by Fig. 1.

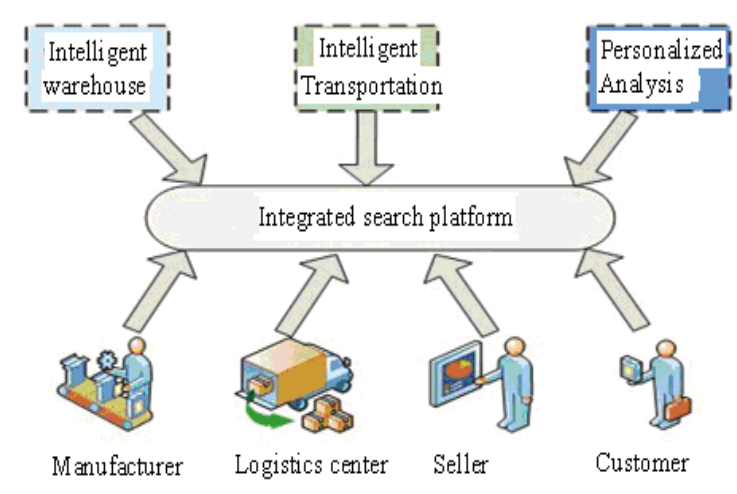

Figure 1. Application of RFID technology in building intelligent logistics system in Internet of things

Intelligent logistics benefits, such as fine-grained, and it is real-time performance, reliability has been reflected in the various services in intelligent logistics. How to configure and use of resources and it is reduce manufacturing cost and enterprises to focus on the problem. To implement this 
strategy, not a highly advanced, reliable and efficient logistics system is unable to realize. With the development of economic globalization and the rise of network economy, the logistics function is no longer simple in order to reduce the cost, but rather becomes improve customer service quality to enhance the comprehensive competitiveness of enterprises. At present, the logistics industry is gradually formed seven development trends, they are respectively and the third party logistics cooperative information, intelligence, environmental protection, enterprise globalization and internationalization, service quality, industry.

\section{Summary}

The paper presents application of RFID technology in building intelligent logistics system in Internet of things. The basic principle of RFID system is the label into the field, RF signal receiving reader, and sending current obtained by the energy product information stored in the chip. The user can be used to prevent or disrupt the tag near the RFID reader is operated through a device to broadcast radio signal.

\section{Acknowledgements}

This paper is supported by Science and technology project of Henan Province (162102310478).

\section{References}

[1] SunYu Jie, Julian Clive. Research of Logistics Product Intelligent System Distribution Based on Internet of Things, JDCTA, Vol. 7, No. 6, pp. $979 \sim$ 986, 2013.

[2] Wei Zhengxi, "Application model of RFID middleware system for manufacturing and services industry", AISS, Vol. 7, No. 2, pp. 168 174, 2015.

[3] Ken Cai. Internet of Things Technology Applied in Field Information Monitoring, AISS, Vol. 4, No. 12, pp. $405 \sim 414,2012$.

[4] Han Xiao, Yuanjiang Li. A New Thought based on the Service Composition of Automatic Transmission Semantic Grid in Internet of Things, IJACT, Vol. 3, No. 7, pp. 10 16, 2011.

[5] Lvqing Yang, Caili Wang, Wenhua Zeng, "Research of Matrix-based Grouping Method on Anti-collision Algorithm for RFID Tag Identification", AISS, Vol. 5, No. 13, pp. 126 133, 2013.

[6] Chenghong Zhang, Tian Lu, Lu Zhang, "Improved Allocation Model of Logistics Service Supply Chain Capability Based on NSGA-II: A Case of Tobacco Group Company", JCIT, Vol. 11, No. 4, pp. $7 \sim 13,2016$.

[7] He Jialiang, Ouyang Dantong, Xu Youjun, Shang Yu. An Efficient RFID Authentication Protocol Supporting Tag Ownership Transfer, IJACT, Vol. 4, No. 4, pp. 244 253, 2012.

[8] Xu Xu, Liu Wei, "Research on Logistics Distribution Center Location Model Based On GIS", JDCTA, Vol. 7, No. 7, pp. $538 \sim$ 545, 2013.

[9] Wei Lifeng, Ji Jianwei, Xu Jiawang. Research on the Production Logistics Information Terminal Based on RFID Technology, JDCTA, Vol. 6, No. 20, pp. 114 121, 2012.

[10] Guo Wang, Dong Dai, "The Novel Model of Configuration and Performance Management System in Internet of Things by RFID and SNMP", JCIT, Vol. 8, No. 4, pp. $618 \sim 626,2013$. 